Rhode Island College

Digital Commons @ RIC

$1-1-2013$

\title{
Predictive Behavioral Cues of Patient Violence in the Emergency Department
}

Jessica A. Roy

Rhode Island College

Follow this and additional works at: https://digitalcommons.ric.edu/etd

Part of the Geriatric Nursing Commons

\section{Recommended Citation}

Roy, Jessica A., "Predictive Behavioral Cues of Patient Violence in the Emergency Department" (2013). Master's Theses, Dissertations, Graduate Research and Major Papers Overview. 201.

https://digitalcommons.ric.edu/etd/201

This Major Paper is brought to you for free and open access by the Master's Theses, Dissertations, Graduate Research and Major Papers at Digital Commons @ RIC. It has been accepted for inclusion in Master's Theses, Dissertations, Graduate Research and Major Papers Overview by an authorized administrator of Digital Commons @ RIC. For more information, please contact digitalcommons@ric.edu. 


\section{PREDICTIVE BEHAVIORAL CUES OF PATIENT VIOLENCE IN THE EMERGENCY DEPARTMENT}

by

Jessica Roy

A Major Paper Submitted in Partial Fulfillment

Of the Requirements for the Degree of

Master of Science in Nursing

in

The School of Nursing

Rhode Island College

2013 


\begin{abstract}
Nurses are often subject to violence at the hands of their patients. The Emergency Department (ED) has become the area most vulnerable in the hospital setting, with contributing factors including the rise of polysubstance drug abuse, the increasing psychiatric population, and overall stressors related to the economy. The ED is the "gateway" to the availability of shelter, medications, and resources for many people that pass through every year. Accurate and timely identification of the most common and easily identifiable precursors of violence is essential in order to develop and implement effective de-escalation techniques that have the potential to reduce actual events. The purpose of this research project was to demonstrate the usefulness of a behavioral cue assessment tool in providing a simple predictor for potential violence in the ED setting. The methodology included a behavioral assessment checklist containing 17 cues developed by Wilkes, Mohan, Luck and Jackson (2010). Nurses completed the 17 item behavioral assessment on all patients being treated in the ED during a three-week time period. All 17 behavioral cues demonstrated a positive predictive factor for violence based on statistical analysis. Given the simplicity, cost effective nature, and predictability, the checklist appears to be feasible to use to potentially reduce healthcare costs related to injuries and emotional distress of nurses at the hands of violent patients. Further research is indicated. This study further exemplifies the qualities of an advanced practice registered nurse (APRN), including research, education, cost containment, and improved patient care.
\end{abstract}




\section{Table of Contents}

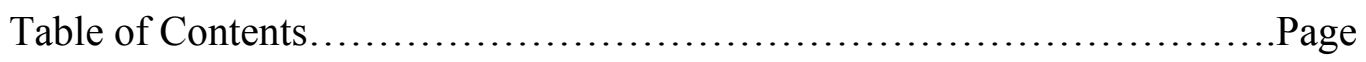

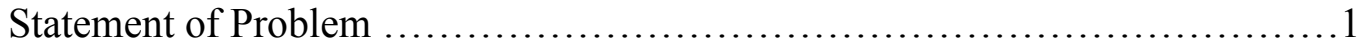

Literature Review....................................................4

Theoretical Framework...............................................13

Methods.............................................................. 16

Results.............................................................. 21

Conclusions and Implications for Advanced Nursing Practice..................26

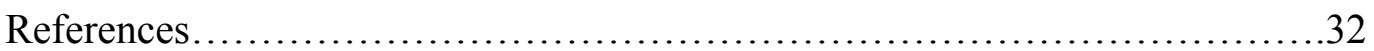

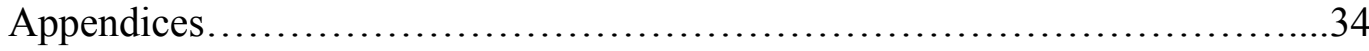


Predictive Behavioral Cues of Patient Violence in the Emergency Department

\section{Statement of Problem}

Workplace violence is a complicated and often underreported occupational hazard that has become almost a social norm in the health care setting (Pich, Hazelton, Sundin, \& Kable, 2010). In 2002, the National Institute for Occupational Safety and Health (NIOSH) defined workplace violence as violent acts (including physical assaults and threats of assaults) directed towards persons at work or on duty. Workplace violence is further defined as physical assault, emotional or verbal abuse, and threatening, harassing or coercive behavior that causes physical or emotional harm (NIOSH, 2002). At particular risk are members of the nursing profession and others with continued patient contact in high anxiety-type situations.

The most common types of physical violence reported by the nurse respondents in a study conducted by the Emergency Nurses Association (ENA) included being “spit on", “hit", "pushed/shoved", "scratched" or "kicked" (Gacki-Smith et al., 2009). Verbal abuse was reported by nearly $70 \%$ of respondents, with the most common examples being "yelled/cursed at", "intimidated" and "harassed with a sexual language/innuendo".

Nurses working in the ED face their own set of individual challenges in dealing with violent patients. Due to the accessibility of the ED, nurses report the highest percentages of abuse and underreporting (Pich et al., 2010). In the ENA study, approximately $25 \%$ of respondents reported experiencing physical violence more than 20 times in the past three years, and almost $20 \%$ reported experiencing verbal abuse more than 200 times during the same period (Gacki-Smith et al., 2009). Multiple factors that 
contributed to the potential for violence in the ED were identified, including alcohol intoxication, drug seeking behavior, polysubstance drug use, overwhelming psychiatric patient population, crowding, prolonged wait times, misconceptions of staff behavior, and poorly enforced visitor policies. Additional causative agents included 24-hour accessibility, minimal security presence, highly stressful environments, anxiety-inducing medical conditions, and access to pharmacy and prescription narcotics.

The broad epidemic of workplace violence, with particular focus on the ED and the nurses that are employed in this setting, is of particular interest nationally. Although patient violence against nurses has been identified in current nursing literature, such as Pich et al., (2010) and Gallant-Roman (2008), as a serious occupational hazard, there is a lack of simple screening tools to identify the potential for violence in this setting (Wilkes et al., 2010). Early recognition of the warning signs of patient violence may reduce the incidence of occurrence or increase preparedness for such an event. Luck, Jackson and Usher (2007) and more currently Wilkes et al. (2010) identified five and 17 behavioral cues, respectively; however there is not a substantial amount of literature testing the usefulness of either of these tools. Kim, Ideker, and Todicheeney-Mannes (2012) discussed the psychometric properties of violence assessment tools and identified the need for such tools to determine overall usefulness as demonstrated by a satisfactory sensitivity, specificity, and inter-rater reliability. Also to improve inter-rate reliability when constructing violence prevention tools, a direct observation of the patients' behaviors is recommended by the authors, since previous studies had pulled information retrospectively from incident reports. Kim et al. (2012) further suggested that an easy-to- 
use violence risk assessment tool would likely identify most of the potentially violent patients without concern for misclassification and prevalence of false positives amongst the sample. Given the ever-expanding problem of patient violence directed against ED nurses and the lack of substantial research regarding a measurement tool, it is clear that further research in this area is warranted.

Next, the literature review will be discussed. 


\section{Literature Review}

A search of the literature was conducted via online databases CINAHL and MEDLINE utilizing the combination of key words "nurse", "nursing", "workplace", "violence" and "emergency department". There were no exclusions to the search. Over 50 articles met some or more of the search criteria and several valuable articles were identified and will be further discussed in a literature review.

\section{Workplace Violence}

Workplace violence has been defined as violent acts, including physical assaults and threats of assaults, directed towards persons at work or on duty. It has been further defined as physical assault, emotional or verbal abuse, and threatening, harassing or coercive behavior that causes physical or emotional harm (NIOSH, 2002). Certain occupations have an obvious potential for violence, such as law enforcement, and these are acknowledged by society as such. There is generally a more infrequent reference to the risk of violence in the health care sector, although the incidence is significantly higher and often underreported (Child \& Mentes, 2010). Gallant-Roman (2008) reported that the cost of workplace violence was estimated to be $\$ 4.2$ billion annually in 2001 . Nurses also reported workplace violence twice as often as any other healthcare provider. The rate of violence against nurses, which was reported as $21.9 \%$, may actually be markedly inaccurate secondary to the lack of reporting, since nurses are four times more likely to encounter violence than any other sector (Gallant-Roman).

The consequences of workplace violence may result in poor retention, emotional distress similar to post traumatic stress disorder, anxiety, impaired work performance, 
and insomnia. There is also evidence that staff members subject to abuse lose confidence and self-esteem and have a higher potential for abuse of sick time, experience burnout, and are more likely to abuse of alcohol and drugs. Rew and Ferns (2005) published suggestions on developing a balanced approach in dealing with violence and aggression in the workplace. Specific recommendations to tackle violent incidents included close circuit television, security guards, addressing the environment, counseling services, poster campaigns, changing room layouts, installing panic buttons, emergency and incident drills and improving waiting areas in facilities. The authors concluded that the amount of violent incidents in the workplace needed to be reduced; areas identified for improvement included adequate reporting, government involvement, and the success of violence prevention programs. Uniquely, the authors also discussed incorporating techniques from other disciplines, including the martial arts, to improve prevention. They specifically suggested incorporating techniques geared at improving communication skills, focusing on trigger factors, and teaching.

Based on a review of the literature, Gallant-Roman (2008) published recommendations that identified strategies to reduce workplace violence. Protection from workplace violence and an unsafe workplace is guaranteed under Occupational Safety and Health Administration (OSHA) regulations and most employee-based groups call for a zero tolerance policy. However, health care workers remain subject to violence at an alarmingly increasing rate. Health care worksites must take an active role in the prevention of workplace violence through prevention programs and interventions. As discussed by Gallant-Roman (2008), workplace violence costs nearly $\$ 4.2$ billion 
annually and nurses were twice as likely to be subject to work-related violence as any other profession. Nurses working in the ED, within mental health facilities, and with the geriatric population were identified as being at particular risk. Based on the review of the literature, Gallant-Roman made recommendations for the success of workplace violence prevention programs including: thorough evaluations of the workplace; utilization of a Top-Down approach; instituting a zero tolerance policy; empowering nurses; and providing education and predicting high risk events.

\section{Violence against Nurses in Emergency Department (ED)}

The most comprehensive study in recent years relating to the incidence of violence against nurses in the ED was conducted by Gacki-Smith et al. (2009). A total of 3,465 registered nurses (RNs) who were members of the ENA participated in the study. The purpose of this study was to identify their own personal experiences with violence, the policies and procedures of their own facility, their beliefs about precipitating factors to violence, and barriers to reporting incidents of violence. A 69 item computerized survey was administered. The most common types of physical and verbal abuse included being spit on, hit, pushed/shoved, scratched, kicked yelled/cursed at, intimidated, and harassed with sexual innuendo. Sixty percent of respondents expressed a safety level at a five or below on a ten point scale, with ten being most safe. Twenty-three percent of respondents were categorized as Frequent Physical Violence Experience (FPVE) nurses, characterized as experiencing violence more than 20 times in the past three years. Twenty percent of nurses were characterized as Frequent Verbal Abuse Experience (FVAE) nurses, in that they were subject to greater than 200 episodes of verbal abuse in a 
three year period. Nurses identified barriers to reporting, including fear of retaliation, ambiguous reporting systems, and the attitude that violence "comes with the job". As the first national study regarding perceptions and private experiences, the study is a major contributor to understanding the present dynamic of patient to nurse violence in an ED setting.

Evidence suggests that the health care industry has been recently identified as one of the most violent workplaces throughout the world. Given that nurses are the health care providers with the majority of the patient contact, they tend to be the workers most commonly abused. Pich et al. (2010) performed a comprehensive review of current literature related to violence against nurses, and further narrowed the review to the ED. One hundred fifty six abstracts were located and 41 met the inclusion criteria: research articles; published in the English language after 2008; studied violence against nurses perpetuated by patients or family members. Although the focus was on the ED, other areas of nursing were included if they were related to the ED. Studies meeting the inclusion criteria included surveys, interviews and qualitative data, and out of 156 abstracts, a total of 41 papers met inclusion criteria. Significant findings included the observation that nurses were often subject to abuse, both verbal and physical, and that that abuse had actually been accepted as part of the job. An overall attitude among nurses that violence had actually become normalized was also revealed. As such, violence becomes a common thread in workplace culture, often making it difficult to develop prevention strategies. Pich et al. (2010) reviewed and identified common risk factors for violence as derived from the literature, which were further structured into 
common themes: history of violence; substance and alcohol abuse; medical diagnosis (particularly mental illness); waiting times; time of day; cultural issues; attitudes of nurses; under-reporting; and management response. Pich et al. (2010) stressed the importance of identifying the obvious risk factors of violence. The warning signs and predictors of violence were also identified, and included a history of violence, substance and alcohol abuse, mental health conditions, those suffering from physical pain, waiting times, time of day (outside of working hours), and cultural diversity. In summary, Pich and colleagues suggest that some nurses have become their own barrier for prevention against violence given their acceptance and normalization of the violence.

Anderson, FitzGerald, and Luck (2010) published an integrative review of interventions to reduce violence against ED. The goals were to minimize the violence directed towards nurses by providing nurses with interventions to counter this violence. Criteria for selection included any research study that discussed a customer- or clientperpetrated violent event toward the person providing care (particularly nurses) and occurring within the ED. Ten quantitative research studies and four reviews were included in this integrated literature review. The authors classified the studies into three major categories of interventions: the workplace environment; workplace practices and policies; and individual/collective skill sets. Anderson and colleagues (2010) concluded that the studies mainly addressed a description of the phenomena of violence against nurses. They alternatively suggested that the focus should actually be the identification of problem solving interventions geared toward the prevention of the phenomenon. Some of the interventions that were examined in the study included metal detectors, staff 
training programs, informational pamphlets and de-escalation kits. No direct intervention demonstrated usability over another. Four studies assessed a single intervention but failed to take into account the context of the violence, and two studies with multiple interventions had difficulties with generalizability. The researchers noted that multi-site and multidisciplinary research studies are urgently needed to promote prevention of violence against nurses in the emergency department. Given the progressive increase in violent events against nurses in EDs, it is also helpful to recognize the strategies that nurses are utilizing in dealing with these aggressive and violent patients.

\section{Specific Strategies to Identify Violent Behaviors in the ED}

Kim et al. (2011) tested the usefulness of an assessment tool for aggressive behavior in the prospective identification of violence in medical surgical units. This research provided the benchmark for testing a simple screening tool for violence. The 17-item checklist, the Aggressive Behavior Risk Assessment Tool (ABRAT), was derived from the published M55 tool, the 5-item STAMP (Luck et al., 2007) and an investigator-developed item. A prospective cohort study was conducted at an acute care hospital in which primary nurses completed a 17-item checklist upon admission. An identical checklist was then completed by a different nurse, in an effort to assess interrater reliability. A separate violent event outcome section was filled out in the event of violence or abuse, or upon discharge, if no abuse had occurred during the patient's admission. A logistic regression model was used to identify the best predictors of violence in this setting. It was reported that 56 patients out of $2063(2.7 \%)$ had one or more violent events. Overall, the staff rated the tool as easy to use. The measure was 
found to have acceptable accuracy, such that it could be useful in the identification of violent patients in medical-surgical units. The main limitation of this study was the inability to generalize to the ED population of patients. Although the ABRAT was derived from a combination of established behavioral cue tools, it was ultimately designed to meet the needs of the medical surgical population only. In contrast, a tool designed for the ED would have cues based on an initial meeting with no previous knowledge of the patient.

Addressing the concept of caring as it relates to nursing, Luck, Jackson, and Usher (2009) conducted a study of 20 RNs designed to identify caring attributes utilized by nurses in the ED to avoid violent events from their patients. This study was undertaken over a five-month period in a 33 bed ED in Australia. The authors utilized a mixed method case study design that included 290 hours of participant observation, 16 semi-structured interviews, and 13 informal field interviews over a five-month period. Five attributes were identified: being safe; being available; being respectful; being supportive; and being responsive. These five attributes were used by nurses to reduce the potential of a violent event and specifically provided a technique that could be implemented if the subjects became abusive or violent. A total of 16 violent incidents occurred despite the strategies employed by the nurses.

Derived from these interviews and observation, Luck et al. (2007) described the five cues of potential violence with an acronym STAMP: Staring and eye contact; Tone and volume of voice; Anxiety; Mumbling; and Pacing. Participants described components or cues in a sequential manner to illustrate the relationship between the 
potential for violence and the number of demonstrated STAMP cues exhibited. Using this technique, a nurse could identify a patient with a potential for violence prior to the actual event. This study was the first that identified observable behavioral cues that related directly to the ED and came directly from patient assessments in that setting. This assessment measure considers the interpersonal and psychosocial aspects unique to the ED and can be applied to patients, visitors, and family members. Given this was one study in one ED, it would be difficult to generalize the findings; however, it could be easily tested in any ED. This research was subsequently utilized in research project conducted by Wilkes et al. (2010).

Lauretta Luck and colleagues Wilkes, Mohan, and Jackson (2010) developed a 17-point behavioral cue checklist deriving from the STAMP study. Wilkes et al. (2010) used a panel of 11 expert nurses and clinicians to initially develop a 37-item questionnaire designed to identify potential cues of patient violence in patients presenting to the ED. These 37 items were composed of 22 items identified by Luck et al. (2007) and 15 additional items were drawn from a literature review. Each of the 37 cues identified were further grouped under the five STAMP components previously identified by Luck et al. (2007). This 37-item questionnaire went through three rounds of Delphi technique in which a series of anonymous questionnaires were sent to a panel of experts. This was followed by analysis and feedback of suggestions in order to come to a consensus. The final product contained 17 total behavioral cues in a checklist form that could be easily observed and visualized without knowing anything about the patient's history. These 17 components can be applied to any ED and included threat of harm, 
aggressive statements, intimidation, clenched fists, resisting healthcare, prolonged staring at nurse, name calling, yelling, increased volume of speech, irritability, walking back and forth to nurses area, pacing room, sharp or caustic retorts, demeaning inflection, belligerence, demanding attention and humiliating remarks. Kim et al. (2012), in a prospective cohort study, revised the 17 point behavioral cues to ten cues (ABRAT), which was identified as being more applicable to the inpatient population. The ABRAT demonstrated $98 \%$ specificity with $43 \%$ sensitivity.

In summary, the literature review established a comprehensive understanding of workplace violence specific to the ED as well as the value of using of a behavioral assessment tool in order to mitigate patient violence. The purpose of this study was to evaluate the usefulness of the 17-point behavioral cue checklist (BCC) developed by Wilkes et al., (2010) in identifying the potential for violence from patients against ED nurses.

The theoretical framework guiding the study will now be discussed. 


\section{Theoretical Framework}

The primary way to stop violence in any form is to prevent it before it begins. This can be achieved through early recognition of the behavioral cues commonly found in those that demonstrate aggression (Luck et al., 2007). The Centers for Disease Control and Prevention $(\mathrm{CDC})$ reported that prevention requires an overall understanding of many factors that ultimately influence violence. The CDC uses a four-level socialecological model that further explains causative agents of violence and how that ultimately affects violence prevention strategies (2012). The CDC's Social-Ecological Model (SEM) framework, which drives the CDC's violence prevention program, also meets the purpose of this research and thus served as a guide for this project.

The SEM model was first developed by Dahlberg and Krug in 2002 as a recommendation for the prevention of violence. In "World Report on Violence and Prevention", the authors stated that this evolving model had been refined further to provide a framework that broadens the understanding of how violent influences differentiate through the model. The SEM is a four-level model that incorporates the individual, the surrounding relationships, the community, and the societal components of the decision making process for each behavior. This model (Figure 1) describes a complex interrelated mixture of influences affecting an individual's behavior.

The individual is the first level and the core of the model. The individual factors are identified as they relate to behaviors. Considerations of the patient's age, education, income, history of substance abuse, history of abuse and personal factors can be identified at this level. 


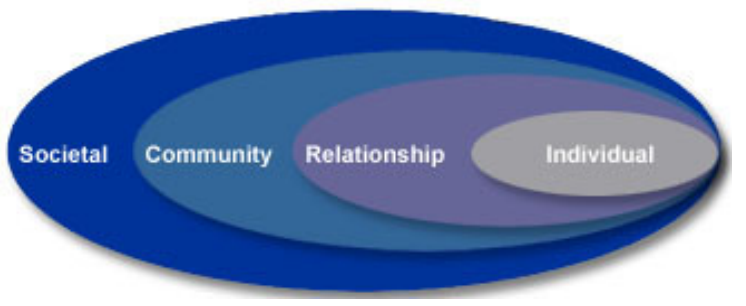

Figure 1. The Social-Ecological Model.

Adapted by the Centers for Disease Control and Prevention from Dahlberg, L. \& Krug E. (2002). Violence: A global public health problem. In J. Mercy, A. Zwi \& R. Lozano (Eds.), World report on violence and health (pp 1-56). Geneva, Switzerland: World Health Organization.

The second level, or the relationship, examines interactions that may be causative agents to potentially increase the risk of experiencing violence according to the CDC. It is important to identify relationships with friends, family, and even health care workers in the setting of the ED as causative factors that affect interpersonal relationships. The third level examines the community and the settings in which the individual works, learns, and lives, and the fourth level addresses the societal factors involved in perpetrating volatile behaviors such as societal policies, economics and overall health (Dahlberg \& Krug, 2002). Prevention strategies can be geared towards all four levels of the SEM.

The proposed behavioral cue assessment research study will be focused on the first two levels of the model, the individual and the relationship. Identifying the individual behavioral cues of a person actually addressed the core of the SEM model. These behavioral cues may also be affected by the relationship between the nurses or other health care providers. Therefore, the pre-identification of behavioral cues may 
overlap into the second tier of the model, the relationship. Since the community and societal components typically affect the individual after discharge from the hospital setting, these areas are not directly affected by the project.

Next, the methods will be presented. 


\section{Methods}

\section{Purpose}

The purpose of this study was to evaluate the usefulness of the 17-point behavioral cue checklist (BCC) developed by Wilkes et al. (2010) in identifying the potential for violence from patients against ED nurses. This study was designed to identify a suspected relationship between the established behavioral cues and the potential for violence.

\section{Research Question}

For RNs working in an ED, can the implementation of a 17-point behavioral cue assessment at first encounter, identify the potential for violence from patients?

\section{Design}

A prospective survey design study was utilized.

\section{Sample}

Inclusion criteria represented all English-speaking adult patients equal to or greater than 18 years of age that presented to the ED during the time period of October 21, 2012 through November 11, 2012. Given the variability of non-verbal cues of pediatric and non-English speaking individuals, these potential subjects were excluded. Site

The Emergency Care Center at Sturdy Memorial Hospital in Attleboro, MA was the site of the data collection. Sturdy Memorial treats 50,000 patients annually through the thirty bed Emergency Care Center and is based in a community setting. 


\section{Measurement}

The BCC (Appendix A) is a 17-point checklist developed by Wilkes and colleagues (2010) in collaboration with Dr. Luck. Electronic permission (Appendix B) was obtained from Luck to utilize the17 point behavioral cue checklist (BCC). Dr. Luck provided the permission for utilization of the 17-point behavioral cue checklist, as she was one of the contributors in the development of the checklist. This behavioral cue checklist was based on her original STAMP model (Luck et al., 2007) and therefore she maintained responsibility for its future utilization. No published research establishing the reliability or validity of the $\mathrm{BCC}$ was found in the literature.

The student researcher, as a seasoned practitioner, identified the potential for variation in interpretation of these cues. The decision was made to provide a definition for each of the 17 behavioral cures. Definitions were based on those found on the Dictionary.com website (http://dictionary.reference.com). These definitions were itemized and copied on to the reverse side of the $\mathrm{BCC}$ for reference by the $\mathrm{RN}$ completing the form if necessary (Appendix C).

The second component of the BCC included the five types of violence, also in checklist format (Appendix A). This section was completed at the time the patient demonstrated any type of abuse or violence and/or at the time of discharge, whichever occurred first. For the purpose of this study, the forms of violence/abuse that were considered were based on the NIOSH (2012) definitions and included actual physical violence, threat of physical violence, verbal threats/harassment/coercion, sexual harassment or no abuse observed. 
Limited demographic information, including the sex and age of the patient and the date of data collection were gathered.

\section{Procedures}

The study was reviewed and ruled exempt by the Institutional Review Board at Sturdy Memorial Hospital. The study was also reviewed and subsequently approved by the Institutional Review Board at Rhode Island College.

Any RN employed in the Emergency Care Center at Sturdy Memorial Hospital was eligible to voluntarily assist with the data collection; no compensation was provided to the collectors. There were no additional responsibilities of the $\mathrm{RN}$ other than completing the $\mathrm{BBC}$ during the routine nursing assessment.

All ED nurses were educated by the student researcher at a monthly staff meeting during a 5-10 minute informational session designed to provide a summary of the project and familiarize them with the data collection tool. Reference materials were made available in the reference cabinet of the ED for clarification of any questions. The education focused on how to complete the 17 point BCC (Appendix A) and also included, on the backside of the checklist (Appendix C), use of definitions of each behavioral cue as identified by the Dictionary.com website

(http://dictionary.reference.com). Nurses who were unable to attend one of the three staff meetings were provided the same training on a one-to-one basis. A total of 62 nurses completed the training. Once training was completed, the checklists were given to the unit secretaries to be placed on each patient's paper chart. ED nurses completed the 17- 
point $\mathrm{BCC}$ on every patient meeting inclusion criteria upon first contact with the patient during the three-week data collection period.

A second nurse also identified as this researcher completed a second assessment at random to evaluate the accuracy of the initial assessment. This was performed in order to address the inter-rater reliability of the tool. This researcher observed the triage interaction between the patient and the primary nurse and completed the additional checklist accordingly. This secondary assessment was completed at the same time the primary nurse was completing the primary assessment. Observation was completed while standing outside the room observing the triage process. The researcher recorded the assessment without any discussion or interaction directly with the primary nurse or the patient being observed. The student researcher completed 30 additional checklists weekly for a total of 90 duplicate assessments. A number was written in the top right hand corner of the checklists that were duplicated to compare during the analysis period.

As part of their routine, the secretaries place any hard copies that are contained within a patient's paper chart into a binder upon completion of the registration process. The BCC was placed on the front of the paper chart when consolidated into a binder by the unit secretaries. These patient charts (binders) were stored in the usual manner, in the numbered slots within each nursing station and were accessed by secretaries, RNs and physicians. The BCC forms were placed in an envelope by the secretaries upon removal of the paper chart from the binder upon transfer, discharge or death of the patient. The envelope of data was collected on a weekly basis by this researcher and was transferred and stored electronically in an EXCEL ${ }^{\mathrm{TM}}$ spreadsheet. 
In the event a violent incident occurred or observation of this behavior, the nurses utilized the Sturdy Memorial Hospital policy and attempted to deescalate using trained techniques. In more severe circumstances, the nurses called a Code 1010 and contacted security for assistance or possible restraint of the individual.

\section{Data Analysis}

Statistics were performed using MedCalc, Version 12.5.0. Descriptive statistics were performed on the study variables. The frequency and percentage of patients demonstrating a violent event was reported. An odds ratio (OR) and confidence interval (CI) were determined for each of the 17 behavioral cues. Inter-rater reliability was calculated based on random audits.

The results will now be discussed. 


\section{Results}

A total of 1808 checklists were completed during the October $21^{\text {st }}$ and November $11^{\text {th }}$ time period in 2012. One hundred and forty seven were excluded secondary to incompleteness or checklists completed on pediatric patients. Out of the remaining 1661 checklists, $1572(94.6 \%)$ confirmed that patients had not exhibited any predictive cues of violence. Of the total number of patients $(\mathrm{N}=1572), 56.4 \%(\mathrm{n}=888)$ were female and $43.5 \%(n=684)$ were male patients. The demographic profile of participants is illustrated in Table 1.

Table 1

Demographic Distribution of Study Participants $(N=1661)$

\begin{tabular}{|c|c|c|}
\hline Age & \% Female & \% Male \\
\hline $18-30$ & 12.9 & 9.4 \\
\hline $31-55$ & 23.9 & 18.7 \\
\hline $56-69$ & 9.2 & 8.1 \\
\hline $70+$ & 10 & 7.5 \\
\hline
\end{tabular}

A total of $42(2.5 \%)$ participants exhibited one of more behavioral cues of violence, but did not exhibit any actual violent episodes. Forty-seven (2.8\%) participants exhibited one or more behavior cues for violence and also exhibited one or more violent episodes. Demographically, the distribution of violence (Table 2) for females and males were $44.7 \%$ and $55.3 \%$ respectively, and $6.4 \%$ of those exhibiting violence were over the age of sixty-nine. The groups most likely to perpetrate a violent event were males aged 31-55 years. 
Table 2.

Demographic Distribution of Violent Patients $(\mathrm{n}=47)$

\begin{tabular}{|c|c|c|}
\hline Age & \% Female & \% Male \\
\hline $18-30$ & 14.9 & 14.9 \\
\hline $31-55$ & 17 & 29.8 \\
\hline $56-69$ & 10.6 & 6.3 \\
\hline $70+$ & 2.1 & 4.3 \\
\hline
\end{tabular}

Of the participants that demonstrated a violent episode $(n=47)$, all exhibited one or more behavioral cues from the checklist: 26 had episodes of verbal abuse $(55.3 \%) ; 11$ threatened physical abuse (23.4\%); eight had an actual episode of physical abuse (17\%); and two sexually harassed staff (4.2\%). (Figure 2$)$

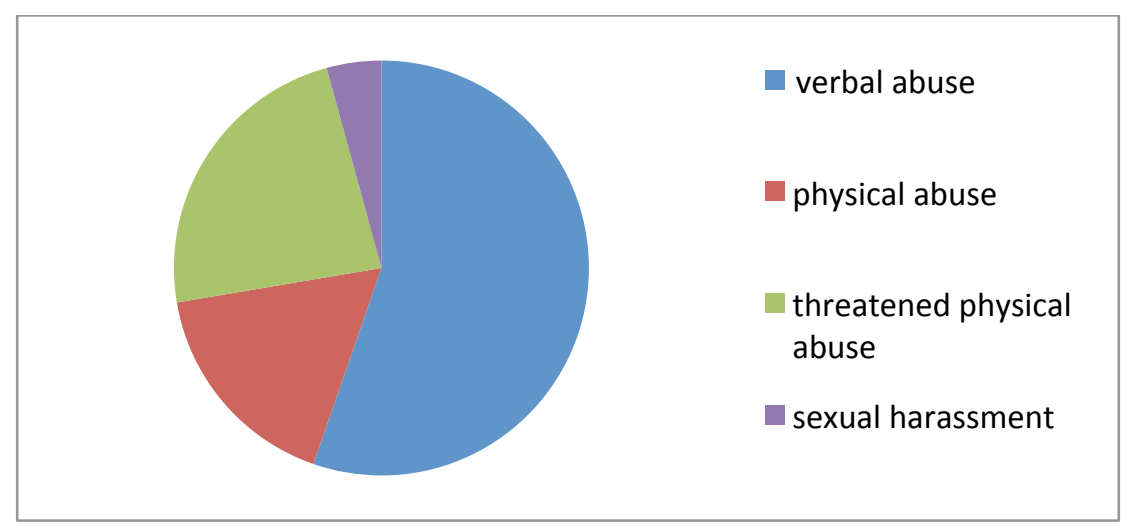

Figure 2. Violent Episodes Breakdown

Some patients had more than one type of violent episode. An analysis of each behavioral cue by frequency and percent of occurrence is illustrated in Table 3. 
Table 3

Analysis of Behavioral Cues: A Comparison of Number and Percentage of Violent vs. Non-Violent Patients

\begin{tabular}{|c|c|c|c|c|}
\hline Behavioral Cue & $\begin{array}{l}\text { \# Exhibited } \\
\text { Violence } \\
\text { (total } n=47)\end{array}$ & $\begin{array}{c}\text { \% Exhibited } \\
\text { Violence }\end{array}$ & $\begin{array}{l}\text { No Exhibited } \\
\text { Violence } \\
\text { (total } n=42 \text { ) }\end{array}$ & $\begin{array}{c}\text { \% No Exhibited } \\
\text { Violence }\end{array}$ \\
\hline Threat of Harm & 17 & 36.1 & 0 & 0 \\
\hline Clenched Fists & 24 & 51 & 4 & 9.5 \\
\hline Name Calling & 12 & 25.5 & 2 & 4.8 \\
\hline Irritability & 40 & 85.1 & 32 & 76.2 \\
\hline Sharp Retorts & 13 & 27.7 & 1 & 2.4 \\
\hline Demanding Attention & 11 & 23.4 & 11 & 26.2 \\
\hline Aggressive Statements & 28 & 59.6 & 2 & 4.8 \\
\hline Resisting Healthcare & 18 & 38.3 & 8 & 19 \\
\hline Swearing & 23 & 48.9 & 2 & 4.8 \\
\hline $\begin{array}{l}\text { Walking Back Forth to } \\
\text { Nurses Station }\end{array}$ & 4 & 8.5 & 0 & 0 \\
\hline Demeaning Inflection & 8 & 17 & 2 & 4.8 \\
\hline Humiliating Remarks & 15 & 31.9 & 2 & 4.8 \\
\hline Intimidation & 10 & 21.3 & 0 & 0 \\
\hline Prolonged Staring & 5 & 10.6 & 2 & 4.8 \\
\hline $\begin{array}{l}\text { Increased Volume } \\
\text { Speech }\end{array}$ & 24 & 51 & 12 & 28.6 \\
\hline Pacing Room & 9 & 19.1 & 6 & 14.3 \\
\hline Belligerence & 15 & 31.9 & 3 & 7.1 \\
\hline
\end{tabular}


Threat of harm, walking back and forth to the nurses' station, and intimidation were exhibited only by the participants that later perpetuated a violent event. Irritability, demanding attention, increased volume of speech, and pacing room were less predictable and were found nearly equally in both violent and non-violent patients. The remaining cues were statistically significant and found most frequently in patients exhibiting violence. This analysis provided a comparison of each behavioral trait as it presented itself within each population and provided an overall occurrence. An odds ratio (OR) and confidence interval (CI) were determined for each of the 17 behavioral cues. The cues are listed in descending order of probability (Table 4).

Table 4

\section{Odds Ratio}

\begin{tabular}{|lcc|}
\hline Behavioral Cues & OR & 95\% Cl \\
\hline Threat of Harm & 48.8 & $2.8-842.6$ \\
Aggressive Statements & 29.5 & $6.4-136.8$ \\
Intimidation & 23.8 & $1.3-420.1$ \\
Swearing & 19.2 & $4.1-88.6$ \\
Sharp Retorts & 15.7 & $1.9-126.0$ \\
Clenched Fists & 9.9 & $3.1-32.2$ \\
Humiliating Remarks & 9.4 & $2.0-44.0$ \\
Walking to Nurses Station & 8.8 & $0.5-168.4$ \\
Name Calling & 6.9 & $1.4-32.8$ \\
Belligerence & 6.1 & $1.6-22.9$ \\
Demeaning Inflection & 4.1 & $0.8-20.5$ \\
Resisting Healthcare & 2.6 & $1.1-6.3$ \\
Increased Volume & 2.6 & $1.0-6.9$ \\
Prolonged Staring & 2.4 & $0.4-12.9$ \\
Irritability & 1.8 & $0.6-5.2$ \\
Pacing Room & 1.4 & $0.5-4.4$ \\
Demanding Attention & 0.86 & $0.3-2.3$ \\
\hline
\end{tabular}

OR=Odds Ratio, $\mathrm{CI}=$ Confidence Interval 
Based on the odds ration, the three most likely traits to predict violent events included threat of harm, aggressive statements, and intimidation. The three least likely traits included irritability, pacing the room and demanding attention. There were no negative predictors of violence from the 17-point checklist.

Approximately $5 \%(n=90)$ of the patients $(\mathrm{N}=1661)$ were assessed directly by this researcher in an effort to address inter-rater reliability and accuracy of the assessments. The agreement percentage was approximately $99.9 \%$ between the two nurses. It was identified by the student researcher that on three separate BCCs a total of four cues were missed, for a percent error of $0.003 \%$. All discrepancies were cues identified by the student researcher and omitted by the primary nurse.

Summary and conclusions will now be discussed. 


\section{Summary and Conclusions}

Workplace violence is a complicated and often underreported occupational hazard that has become almost a social norm in the health care setting (Pich et al., 2010). Workplace violence has been defined as violent acts, including physical assaults and threats of assaults, directed towards persons at work or on duty. It has been further defined as physical assault, emotional or verbal abuse, and threatening, harassing or coercive behavior that causes physical or emotional harm (NIOSH, 2002). Nurses working in the ED face their own set of individual challenges in dealing with violent patients. Due to the accessibility of the ED, nurses report the highest percentages of abuse and underreporting (Pich et al.).

The purpose of this study was to evaluate the usefulness of the 17-point behavioral cue checklist (BCC) developed by Wilkes et al. (2010) in identifying the potential for violence from patients against ED nurses. The CDC's Social-Ecological Model (SEM) framework (CDC, 2012), first developed by Dahlberg and Krug (2002), served as the framework that guided this project. The RNs that participated in the study were trained and collected the data at Sturdy Memorial Hospital by completing the BCC on all patients' meeting inclusion criteria. The first half of the collection data was obtained at time of triage and the second component at time of discharge or any demonstration of patient violence. Inter-rater reliability was also addressed through duplicate checklists completed by the student researcher.

During the three-week collection period, 1808 checklists were completed. One hundred and forty seven checklists were excluded secondary to incompleteness or having 
been completed on a pediatric patient. The total distribution of patients included $56.4 \%$ female and $43.5 \%$ male with the largest representation of patients falling into the $31-55$ years of age demographic. Most patients did not exhibit any behavioral cue of violence (94.6\%). There were a total of 42 patients that exhibited one or more behavioral cues but did not actually demonstrate any violence. Forty seven patients exhibited both violence and at least one behavioral cue of violence. The demographic distribution of violent patients was $55.3 \%$ male and $44.7 \%$ female. The four types of violence were distributed as follows: verbal abuse $55.3 \%$, physical abuse (17\%), threatened physical abuse (23.4\%) and sexual harassment (4.2\%). Additionally, each of the 17 behavioral cues was analyzed comparing the probability of each cue as exhibited by the violent patients versus non-violent participants. Threat of harm, walking back and forth to the nurses' station, and intimidation were cues only exhibited by violent patients. Proportionately, clenched fists, name calling, sharp retorts, aggressive statements, resisting healthcare, swearing, demeaning inflection, humiliating remarks, prolonged staring, and belligerence were all statistically more significantly found in patients that exhibited violence. Irritability, demanding attention, increased volume of speech and pacing room were less predictable and nearly equally found in both violent and non-violent patients. In addition to the probability comparison, the odds ratio and confidence interval were also determined to substantiate the predictability of each behavioral cue relating directly to violent events. The overall frequency of violence was comparable to published literature, at $2.8 \%$ total subjects demonstrating a violent event. Inter-rater reliability was determined to be approximately $99.9 \%$ with the most commonly omitted behavioral cue being irritability. 
All 17 behavioral cues found on the $\mathrm{BCC}$ were positive predictors for violence against nurses in the ED.

There were a few significant limitations to this study, including primarily the voluntary nature of the data collection, not knowing which RNs actually participated and which ones did not. Second, there were omissions (nearly $10 \%$ ) in completion of the second component of the checklist, rendering these particular checklists inadmissible to the study. Incomplete checklists and missing data may have skewed the results of the study. Additional training, stressing the importance of completion of the second half of the questionnaire, may have improved compliance. Another option might have been to request that the secretaries audit the checklists and remind the discharging RNs to complete both parts of the BCC. Also, although significant data was ultimately obtained, the collection period was only a three-week time span at a single site, in a community hospital setting, which does not promote generalizability. Additional test periods would be recommended at multiple sites during random times throughout the year to mitigate this limitation. It may have been helpful to collect additional data about patient demographics, including ethnicity and documentation of the patient's significant medical and psychiatric conditions. An open ended qualitative question regarding nursing opinion in regards to the tool may have been helpful in verifying its overall usefulness.

Based on this work, it is concluded that the BBC checklist (Wilkes et al., 2010) may be an appropriate tool for use in the ED setting to pre-identify the potentially violent patient. It is simple to use, requires minimal training, and has been demonstrated to appropriately identify patients with a potential for violence. A recommendation will be 
made to implement this tool in the ED, based on this research, as a written or computerized assessment. Further education should be considered in the handling and management of actual and potentially violent patients. Skills workshops should be offered to teach RNs techniques to deal with potentially violent individuals and in collaboration with the interdisciplinary team, including Nurse Practitioners and Physicians, to identify how to properly medicate and treat these potentially difficult patients.

Next, recommendations and implications will be discussed. 


\section{Recommendations and Implications}

A primary recommendation for practice is to implement the $\mathrm{BBC}$ checklist

(Wilkes et al., 2010) in EDs. This checklist is relatively quick and easy to use and could be implemented in paper or computerized form with minimal disruption to routine nursing practice in the ED. Key organization such as the Emergency Nurses Association (ENA) could be extremely influential in advocating the implementation of a violence risk assessment in the ED.

This research project has established a foundation for further teaching opportunities as it relates to violence against nurses in the health care setting. Basic educational programs need to raise awareness of this issue and include assessment and management strategies that nurses can use across settings. Education regarding treatment of these potentially violent patients and techniques to appropriately treat or deescalate them is crucial to the success of preventing violence, given this assessment tool simply pre-identifies and relies on the nursing staff to attempt mitigation of this violence. Advanced Practice Registered Nurses (APRNs), including Acute Care Nurse Practitioners (ACNPs), need to seize teaching opportunities related to violence assessment and prevention and maintain awareness at the forefront of optimal practice. Educating both nursing and the interdisciplinary team members as to how to safely and effectively manage violent individuals once identified through the assessment tool is critical. A Violence Prevention Program could empower RNs to improve recognition of violence and development of useful techniques to reduce the incidence in the ED and other health care settings. Program development is a potential area for APRNs and 
education in the prevention of workplace violence is a continued up-and-coming area for advancement.

The APRN has the training needed to implement and evaluate evidenced based projects. This work has opened the door to many additional topics for exploration in this area of study including designing, implementing, and evaluating interventions to manage violent patients and techniques for prevention. Also a significant potential area for study remains identification of the causes for such human behavior. A study that involves violent participants and post violence counseling would also be an interesting area for future research.

Violence in general and workplace violence in particular is of central importance to nursing but also to the nation overall. Violence is a rapidly escalating social issue that threatens the well-being and quality of life of all Americans. APRNs can be influential in this important area of clinical prevention and population health. Lobbying at the local, state, and national level is critical. The APRN role includes advocating for a zero tolerance policy in regard to violence against nurses. Acting as a role model and promoting an environment without judgment or fear of retaliation in relation to reporting such violence is a key component. Care should also be taken to educate colleagues and fellow nurses against normalizing subtle and outward cues of violence. Lobbying for consistent and appropriate repercussions for those that perpetuate violence against nurses and seeking treatment for rehabilitation is indicated. Encouraging lawmakers to initiate, expand, and maintain laws that protect nurses while at work and promoting accurate and timely reporting of such incidents is a must. 
In summary the APRN role is for advancement in the practice of nursing through education and evidenced based research. APRN responsibilities are broad yet crucial in the promotion of national health and safety. This safety starts with the nurses and can be delivered with compassion and caring, as an accurate representation of the profession of nursing. 


\section{References}

Anderson, L., FitzGerald, M., and Luck, L. (2010). An integrative literature review of interventions to reduce violence against emergency department nurses. Journal of Clinical Nursing, 19, 2520-2530. doi:10.1111/j.1365-2702.2009.03144.x

Centers for Disease Control and Prevention. (2012). The Social-Ecological Model: a framework for prevention. Retrieved from http://www.cdc.gov/ViolencePrevention/overview/social-ecologicalmodel.html

Child, R. \& Mentes, J. (2010). Violence against women: the phenomenon of workplace violence against nurses. Issues in Mental Health Nursing, 31, 89-95. doi: $10.3109 / 01612840903267638$

Dahlberg, L., and Krug, E. (2002). Violence: A global public health problem. In J. Mercy, A. Zwi, \& R. Lozano (Eds.), World report on violence and health (, pp. 156). Geneva, Switzerland: World Health Organization.

Gacki-Smith, J., Juarez, A., Boyett, L., Homeyer, C., Robinson, L., \& MacLean, S. (2009, July/August). Violence against nurses working in US emergency departments. The Journal of Nursing Administration, 39, 7/8, 340-349.

Gallant-Roman, M. (2008). Strategies and tools to reduce workplace violence. $A A O H N$ Journal. 56(11), 449-454.

Kim, S., Ideker, K., and Todicheeney-Mannes, D. (2012). Usefulness of aggressive behaviour risk assessment tool for prospectively identifying violent patients in medical and surgical units. Journal of Advanced Nursing, 68(2), 349-357. doi:10.1111/j.1365-2648.2011.05744.x 
Luck, L., Jackson, D., and Usher, K. (2007). STAMP: components of observable behaviour that indicate potential for patient violence in emergency departments. Journal of Advanced Nursing, 59(1), 11-19. doi:10.1111/j.13652648.2007.04308.x

Luck, L., Jackson, D., and Usher, K. (2009). Conveying caring: Nurse attributes to avert violence in the ED. International Journal of Nursing Practice, 15, 205-212. doi:10.1111/j.1440-172x.2009.01749.x

National Institute for Occupational Safety and Health. (2002). Violence: Occupational hazards in hospitals. Retrieved from http://www.cdc.gov//niosh/docs/2002101/pdfs/2002-101.pdf

Pich, J., Hazelton, M., Sundin, D., and Kable, A. (2010). Patient-related violence against emergency department nurses. Nursing and Health Sciences, 12, 268-274. doi:10.1111/j.1442-2018.2010.00525.x

Rew, M. and Ferns, T. (2005). A balanced approach to dealing with violence and aggression at work. British Journal of Nursing, 14(4), 227-232.

Wilkes, L., Mohan, S., Luck, L., and Jackson, D. (2010). Development of a violence tool in the emergency hospital setting. Nurse Researcher, 17(4), 70-82. 


\section{Appendix A.}

Data Collection Form

Patient Age

Sex

Date:

1. Threat of Harm

2. Clenched fists/Tensed Posture

3. Name Calling

4. Irritability

5. Sharp or Caustic Retorts

6. Demanding Attention

12. Humiliating Remarks
7. Aggressive Statements

8. Resisting Healthcare

9. Swearing

10. Walking back and

forth to nurses station

11. Demeaning Inflection

17. Belligerence
14. Prolonged Staring

at Nurse

15. Increased volume

of Speech

16. Pacing Room

9

Upon initial assessment of each patient please circle any/all behavioral cues exhibited by the patient. EXCLUDE pediatrics \& non-English speaking individuals.

13. Intimidation

Upon discharge of patient or at the time of any violent event, please identify.

Actual Physical Violence

Threat of physical violence

Verbal threats/Harassment/Coercion

Sexual Harassment

No abuse 


\section{Appendix B.}

\section{Permission to Use Behavioral Cue}

From: Lauretta Luck <Lauretta.Luck@uws.edu.au>

To: Roy, Jessica A. <jthibeault_8033@email.ric.edu>

Subject: RE: Permission to include STAMP in research study

Date: Tue, Jun 26, 2012 3:27 am

Dear Jessica,

Thank you for your email and your support of my work. Please feel very welcome to use any of my published works to help inform your studies. It is a pleasure for me to see my international colleagues finding this relevant and useful.

Please feel very welcome to contact me if I can help further. I hope you enjoy your studies and all goes well.

Kind Regards,

Dr Lauretta Luck | Senior Lecturer \& Director Academic Program Curriculum

School of Nursing and Midwifery | University of Western Sydney

Building EBLG Room 31, Parramatta South Campus

P: 96859568 |F: 96859599 | E: Lauretta.Luck@uws.edu.at

www.uws.edu.au/nursing

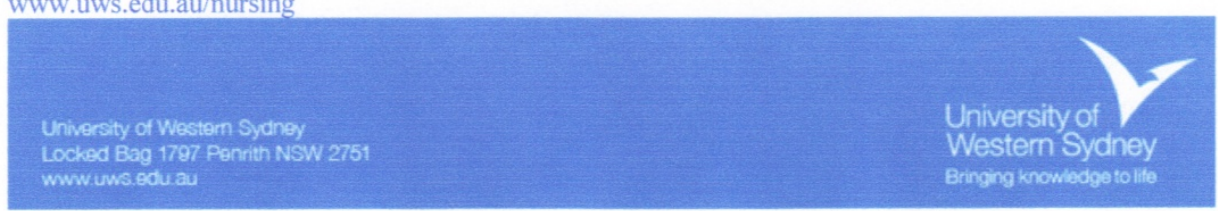

This message contains information that may be confidential and privileged. Unless you are the addressee (or authorised to receive the message for the addressee), you may not use,

copy or disclose to anyone the message or any information contained in the message. If you have received the message in error, please advise the sender by return email and delete the message.

From: Roy, Jessica A. [mailto:jthibeault_8033@email.ric.edu]

Sent: Tuesday, 26 June 2012 7:06 AM

To: Lauretta Luck

Subject: Permission to include STAMP in research study

Dr. Luck,

I am conducting a Masters Project in Nursing and would like to test some of the most prevalent behavioral cues as they relate to violence of patients in the ER. I have read many of your research projects in publication and would appreciate your permission to include some of the components from your "STAMP" tool and your more recent project published in Nurse

Researcher in 2010. I am requesting your permission to include some of these behavioural cues in my research project. Your timely response is appreciated. Best of luck with further research studies!

Kind Regards

Jessica Roy RN BSN 


\section{Appendix $C$.}

\section{Definitions}

1. Threat of Harm- Any expression or intent (verbal or nonverbal) to inflict hurt (mental of physical) onto another person

2. Clenched Fists/Tensed Posture- Rigid arms, does not appear comfortable

3. Name Calling-Any offensive name designed to induce an emotional response from receiver

4. Irritability-Quickly irritated or easily annoyed, sighs when interrogated etc.

5. Sharp of Caustic Retorts- Responds quickly, sharply or angrily in response

6. Demanding Attention- Requiring or asking for more care than what is generally due for the particular situation

7. Aggressive Statements- Taking action without provocation, statements that are attacking in nature

8. Resisting Healthcare- Generally not in accordance with recommended procedures or refuses treatments ie. Vital signs, labs, x-ray etc.

9. Swearing- Uses curse words in routine verbal communication with visitors or staff

10. Walking back and forth to nursing station- Excessive ambulation to the nurse's station without significant reason

11. Demeaning Inflection- Reduce in worth or character to degrade others

12. Humiliating Remarks- To damage someone's dignity or pride, generally publically in an attempt to embarrass

13. Intimidation- To fill another with fear by force of domination

14. Prolonged Staring at Nurse- More than appropriate eye contact

15. Increased Volume of Speech-Raising voice in order to drown out others or yelling

16. Pacing Room- Unable to remain calm in stretcher or chair

17. Belligerence- Quality of being hostile, demonstrating an aggressive demeanor 